\title{
Contralateral suppressive fields of binocular combination'
}

\section{Abstract}

Contours viewed by one eye produce zones of suppression in the contralateral eye's view. The possible functional significance of such contralateral suppressive fields (CSFs) for maintaining convergence-fixation, and for stereoscopic "fusion," is discussed.

\section{Introduction}

If one eye views a horizontal band, and the other a vertical one, the combined view is not a simple cross; instead, each black contour "carries with it" a portion of its white field (Fig. 1a). If lines in one view cross lines in the other, one line disappears near the intersection (Fig. 1b). Kaufman (1964) argues that such

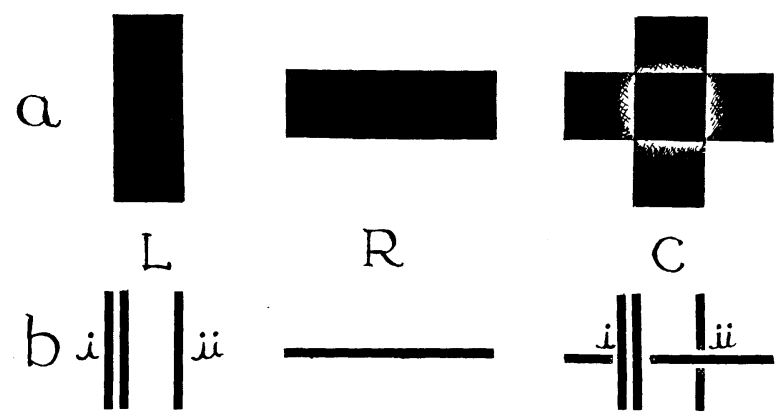

Fig. 1. Stereograms illustrating contour-bound suppression. In each case, $L$ is the left half-view, $R$ is the right half-view, $\mathrm{C}$ is the combined view. At a: each bar's contour carries a halo of its ground into the combined view (Panum, 1858). At b: at the intersection of contralateral lines in the combined view, one or the other disappears. disappearing lines show a suppression of one contour by the other, spreading from their point of intersection. I propose instead that any contour that falls in one eye is surrounded by a zone of some finite size, in the combined field of view, within which all contributions of the other eye are suppressed.

\section{Purpose}

To demonstrate contralateral suppression of nonintersecting contours.

\section{Method}

Twelve Ss viewed the stereogram of Fig. 2 in a Zeiss stereoscope, describing the appearances of "stripes" $a$ and $b$ (whose widths were based on preliminary study with four other Ss). Stripe b subtends $15 \mathrm{~min}$ of visual angle; both its top and bottom contours were simultaneously visible to all Ss. A stripe of the same width, a, should be produced by contours i (left view) and ii (right view), but no S reported seeing a stripe with clear top and bottom contours: either i or ii could be detected at any place at any time, but not both.

\section{Diseussion}

Contours i and ii each produce contralateral suppressive fields (CSFs), preventing their simultaneous projection into the combined view. The vertical extent of a CSF can be assigned a preliminary value of approximately $15 \mathrm{~min}$. To measure the horizontal size of a CSF directly would require contralaterally-viewed vertical contours, and convergent eye-movements would inevitably contaminate the results. In Kaufman's study (1964), the suppression of a horizontal line in one eye by two verticals in the other (Fig. 1b, i) offers indirect evidence that the CSF is about 14 min in horizontal extent, as well.

These estimates are only approximate, however, be-

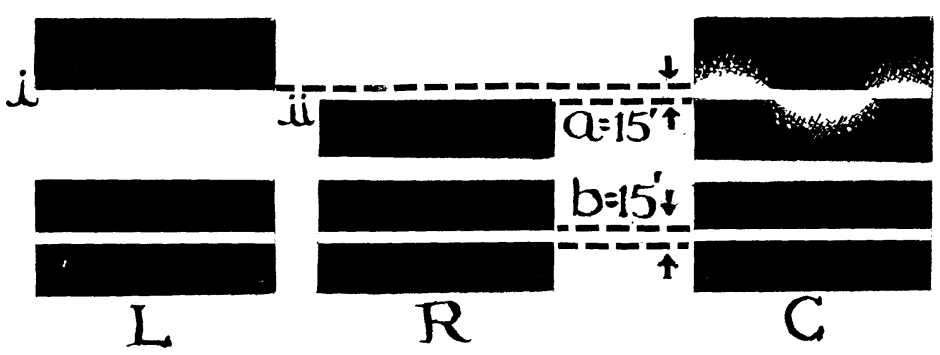

Fig. 2. Stereogram demonstrating that non-intersecting contours (i in the left half-view, ii in the right half-view) carry contralateral suppressive fields in the combined view (C). 

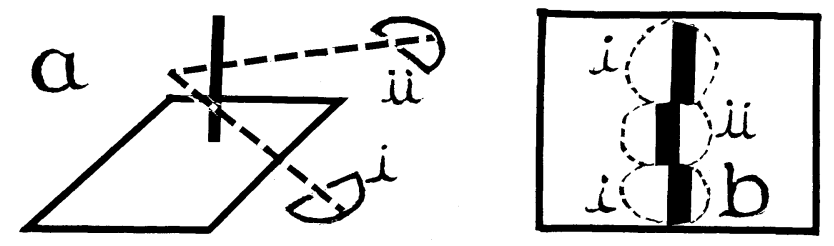

Fig. 3. The effect of slight convergence errors (at a) would be to produce misalignment of the contour fragments in the zones contributed by each eye (i, ii) to the combined view (at $b)$.

cause differences in contour geometry affect the strength of the suppressive halo: e. g., a dot readily suppresses a line of equal visual subtense, and a thin line suppresses a band; such variations might be explained if the CSFs generated by each unit length of contour (or contourreceptive retinal field) summate to produce the effective strength of the resultant zone of suppression. Shapesampling is needed to test this hypothesis, and subjectsampling is needed to determine the range of CSF acuities.

A possible function of contralateral suppression is suggested in Fig. 3: Objects that lie slightly nearer or farther than the point of convergent fixation (or horopter) project two sets of contours in the combined view; if two contralateral contours fall within each others' CSFs, the piecemeal alternation-of-suppression of the contours would reveal their misalignment and provide a precise index (i. e., vernier acuity) of the convergence error. Furthermore, alternate suppression of nearby contours might yield apparent fusion (i. e., achieve "'phenomenal identity") simply as a result of stroboscopic motion. If this latter view is valid, preventing the binocular rivalry would prevent the "fusion" and, perhaps, destroy the stereoscopic depth effect, itself. This possibility is presently being investigated.

\section{References}

KAUFMAN, L. On the spread of suppression and binocular rivalry. Vision Res., in press.

PANUM, P. Physiologische Untersuchungen uber das Sehen mit zwei Augen. Kiel: Schwersche Buchhandlung, 1858).

\section{Note}

1. Supported in part by grant GB-71 from the National Science Foundation. 